\title{
Lenguaje verbal desplegado, lenguaje sintético. Estudio de las interacciones verbales en las clases de Educación Física
}

\author{
Expanded language, reduced language. Study on the verbal interactions at Physical Education class
}

\author{
Maria Victoria Goicoechea Gaona \\ Centro Regional Universitario Bariloche / Universidad \\ Nacional del Comahue, Argentina \\ marivi.goico@gmail.com
}

\section{Resumen:}

Se presenta un aspecto de los resultados obtenidos en un trabajo de investigación realizado en el marco del programa de doctorado: "Bases psicológicas y actividad físico-deportiva: Acción y desarrollo" del Departamento de Ciencias de la Educación de la Universidad de La Rioja (España).

Desde el Interaccionismo sociodiscursivo (Bronckart, 2004) y la teoría de la actividad (Leontiev, 1983) se han estudiado las interacciones verbales en las clases de Educación Física. Se observaron sesiones completas con el fin de registrar y analizar las acciones verbales desplegadas por los profesores y el efecto en las acciones generales (no verbales) de los alumnos. Fundamentalmente, se expondrán los aspectos que consideramos más relevantes acerca de la incidencia del lenguaje (las palabras del profesor) en las acciones motrices que los alumnos realizan. Las clases de Educación Física observadas presentan particulares respecto del uso del lenguaje que hemos dado en denominar: lenguaje sintético y lenguaje desplegado.

El propósito es aportar, desde una mirada externa a la Educación Física, dado que la metodología de análisis proviene del área de conocimiento de la Didáctica de la lengua, información acerca del papel fundamental que tiene el lenguaje verbal en la Educación Física.

PaLABras CLAVE: Interacción, Acciones verbales, Acciones generales, Lenguaje sintético, Lenguaje desplegado.

\section{Abstract:}

This paper zooms in on an aspect of research findings from a thesis study framed within the doctorate program "Psychological foundation behind sporting and physical activity: action and development," Education Sciences Department of La Rioja University, Spain.

From a combined perspective between Social Interactionism (Bronckart, 2004) and Activity Theory (Leontiev, 1983), studies were performed on the verbal interactions registered in Physical Education classes. Observations were carried out in order to record and analyze the verbal actions displayed by teachers and their effect on the student's general (not verbal) actions. Mainly, the focus will be placed on what we deem are relevant aspects of the language effect (i.e. literal words from the teacher) on the student's physical actions. The PE classes we observed show distinctive characteristics regarding language use, which will be referred to as reduced language and expanded language. The purpose is to work from a perspective external to PE, given that the analysis methodology stems from the Language Didactics knowledge area, and provide information about the fundamental role of verbal language in PE.

KEYWORDS: Interaction, Verbal action, General action, Reduced language, Expanded language.

\section{INTRODUCCIÓN}

Con el objetivo de contribuir al estudio del uso del lenguaje (comunicación verbal) en las clases de Educación Física, se observaron y analizaron un total de 40 clases de nivel primario y secundario. Trabajo realizado en el marco del interaccionismo sociodiscursivo (en adelante ISD) y en la línea de investigación conocida como análisis de las consignas de tareas o análisis de la actividad de comunicación de los textos de las clases (Riestra, 2000, 2004 y 2008). En Argentina en el marco de la línea de investigación mencionada se están desarrollando instrumentos metodológicos para el estudio del diálogo en las clases (Riestra, 2019, Goicoechea, 2019).

Partimos de la concepción de que la interacción es fundamental en el desarrollo humano, postulada por el ISD. Mediante la ejecución de acciones los seres humanos interactuamos con el medio. Toda acción está 
situada en un contexto determinado, es promovida por una necesidad y realizada por un agente particular. En consecuencia, nos propusimos abordar las acciones de lenguaje producidas en el marco de la actividad de enseñanza con el fin de estudiar la forma en que están constituidos los enunciados y develar el modo en que las acciones generales determinan las acciones verbales del profesor.

Estudios previos (Goicoechea, 2015, Goicoechea y Goicoechea, 2017) nos han llegado a considerar que en las clases de Educación Física es posible observar la relación entre las acciones verbales y las acciones generales, así como la estrecha relación que existe entre las condiciones en las que se produce la interacción (los elementos extraverbales) y el sentido (la comprensión) de los enunciados. El análisis de las consignas ha documentado que el diálogo de la clase mediado por la realización de las acciones generales (motrices, en el caso que nos ocupa) es infinitamente más rico e ilustrativo que en las clases de otras áreas.

\section{MARCo TEÓRICo}

Nos planteamos el estudio de los textos como producto de las acciones verbales en la interacción docentealumnos en situación de clase para examinar la relación entre las formas lingüísticas que conforman los textos y la finalidad que con ellos se pretende. En un intento de comprender las relaciones entre las acciones humanas generales y las acciones semiotizadas (acciones verbales), centraremos el análisis en los tipos discursivos, es decir, en los "formatos accionales de lenguaje" que Bronckart conceptualiza como herramientas socialmente construidas que los hablantes utilizamos para pensar (Bronckart \& Stroumza, 2003).

En tal sentido, para elaborar los enunciados con los que interactuamos, los hablantes ponemos en juego los recursos lingüísticos que nuestra lengua nos proporciona y los organizamos según combinaciones particularmente heterogéneas a las que Bronckart (2004) denomina tipos discursivos. Dicho de otro modo, los textos están compuestos por configuraciones de unidades lingüísticas (sub-conjuntos de tiempos verbales, de pronombres, de organizadores textuales, de adverbios, de modalizaciones, etc.) y de modos de organización sintáctica relativamente estables.

Los tipos discursivos son cuatro y surgen de la combinación de operaciones de lenguaje que un hablante realiza según el uso que precise. Operaciones relacionadas, básicamente, con dos aspectos: por un lado, el modo de representación del contenido referencial movilizado en el texto, o sea cómo organizamos el contenido temático verbalizado en nuestros enunciados: en el orden del contar o del exponer y, por otro lado, las referencias a la situación material de la enunciación, es decir, si en el texto se alude a las relaciones (implicación o distancia) que se establecen entre los protagonistas de la enunciación: el agente y los destinatarios, y respecto de otros personajes citados en el texto (voces).

De los desarrollos sobre el habla dialogal realizados por Jakubinskij (2018) tomamos la noción de estereotipos verbales. El autor estudió la correspondencia que existe entre los estereotipos de la vida cotidiana (o sea, ciertas situaciones contextuales que se repiten) y los estereotipos verbales. Entre estos últimos ubica las fórmulas de saludo, las referencias a temas de moda en un período determinado, etc., y afirma que: "a determinados períodos del día corresponden determinados hechos verbales” (Jakubinskij, 2018, p.78). Se trata "del uso de frases listas, ya construidas, es decir, de construcciones en el sentido sustantivo de esa palabra” (Jakubinskij, 2018, p. 82).

En el mismo sentido, afirma que en el transcurso de la comunicación, particularmente en el diálogo, el entorno cotidiano es uno de los factores de percepción del habla, uno de los elementos portadores de información. El propio proceso del habla se apoya inconscientemente en el papel que juega el entorno cotidiano en la comunicación, por lo que el proceso del habla está relacionado con los estereotipos de la vida cotidiana, lo que genera "bloques de frases estereotipadas, ligadas de alguna manera a determinadas situaciones de la vida cotidiana y a temas estereotipados de conversación” (Jakubinskij, 2018, p. 81). La comprensión depende tanto o más de la circunstancia en la que transcurre la interacción que del significado de las palabras empleadas. 
IEn síntesis, el enunciado es el resultado de la actividad común del locutor y el interlocutor, del fenómeno de la percepción. Es decir, de la propiedad de anticipación de respuesta que cada enunciado supone, de la respuesta interna que implica y del papel de las frases cliché o estereotipos que hacen posible la comprensión cuasi tácita del enunciado (gesto, mímica, entonación) "cuando los interlocutores se comprenden con pocas palabras en una situación estándar” (Ivanova, 2010: 49).

\section{Metodología}

En el marco teórico al que adherimos, los enunciados registrados en situación de clases constituyen una herramienta para analizar el flujo de la acción, es decir, la actividad realizándose.

Por ello se planteó la observación puntual de clases completas de Educación Física. Para que la muestra fuera representativa, se seleccionaron diferentes centros educativos de la ciudad de San Carlos de Bariloche (Río Negro-Argentina), priorizando las escuelas públicas y abarcando la totalidad de los grados en los que se organiza la educación obligatoria.

Se diseñó un estudio multidimensional para el registro de dos modalidades de interacción:

- verbal, a través de los enunciados de las consignas y de los enunciados con los que los alumnos intervienen en las clases;

- no verbal (acciones generales, Bronckart, 2007), categoría en la que se incluyen las conductas relacionadas con los movimientos corporales y la disposición en el espacio propios de la disciplina.

Se procedió a la observación no estructurada de registros naturales. El material que se obtuvo fue el producto de la actividad verbal, oral, de los profesores de Educación Física. Para el análisis, los textos orales de la clase fueron transformados en textos escritos, según dos modalidades de registro: el natural o descriptivo (al que corresponde la mayor parte del texto) y el narrativo. El último consiste en incluir, entre paréntesis, información breve sobre las acciones no verbales del profesor y de los alumnos. En algunos casos para una mejor comprensión, se incluyó el dibujo de esquemas que representan los movimientos o los desplazamientos efectuados por los alumnos.

Se realizaron dos tipos de análisis. En primer lugar, el Análisis de la actividad de comunicación de los textos de las clases (Riestra, 2000, 2004 y 2008) que consiste en identificar los momentos de la actividad de la clases y las consignas de tareas formuladas en cada uno de ellos. A partir de las consignas, es posible determinar las tareas, las acciones que el profesor explicita para la ejecución de las tareas, y las operaciones supuestas.

El diálogo de la clase evidencia la dinámica de la actividad. La categoría de análisis que hemos denominado Modalidad de la interacción y las subcategorías que la conforman, hacen posible la aprehensión de la actividad (Leontiev, 1983).

Modalidad de la interacción:

- Formulación de la consigna (consigna propiamente dicha).

- Repeticiones.

- Ampliación de la tarea (nuevos enunciados que completan la consigna inicial).

- Modificaciones o reformulación de la tarea (enunciados que introducen cambios para corregir la consigna inicial).

El sistema de categorías implementado documenta el proceso por el cual se realiza la actividad de la clase. En segundo lugar, el Análisis textual de los enunciados de las clases basado en los tres niveles de organización que conforman los textos, y que Bronckart (2004) dio en llamar la arquitectura textual:

- Nivel de la infraestructura: Tipos discursivos (Discurso interactivo, Discurso teórico, Narración y Relato interactivo). 
- Nivel de los mecanismos de textualización: Sintaxis propia del diálogo (rupturas, cambios bruscos, simplificación, etc.)

- Nivel de los mecanismos de enunciación: Voces.

Los datos que se desprenden del análisis textual nos permiten documentar cómo los estereotipos verbales (clichés) hacen posible y dinamizan la comunicación en las clases. El análisis textual descontextualizado de la actividad no hubiera sido suficiente para captar el sentido de las palabras del profesor ni para determinar los resultados que a continuación se exponen.

\section{Resultados: Articulación entre las acciones Verbales y las generales}

Las particularidades del lugar de la clase (gimnasio) y de las tareas, la presencia de elementos (conos) y la precisión con la que han de ser ubicados, y el trabajo en equipo, entre otras, son características propias del área que dan una impronta particular a los enunciados:

D: Todos contra la pared (los alumnos hablan a la docente del juego que acaban de realizar, mientras ella los ubica).

D: Miren, les voy a contar: Cuando los toco se quedan congelados con cara de espantados (los alumnos están en fila, junto a la pared, ella los va tocando y los chicos hacen muecas forzadas. La docente comenta y celebra las caras).

D: Les voy a dar un número (interrumpe la explicación para comentar las caras de los niños). Algunos geniales: mirá a Andrea. Relajamos las caras.

D: El número 1 se para acá, el 2 acá; fila india (la interrumpen). Todavía no di los números... (Termina de asignar un número a cada alumno y coloca seis conos a cierta distancia de los niños, sobre la línea amarilla).

D: Uno, al cono 1; dos, al cono 2, tres al cono...

(Se forman seis filas de niños detrás de cada cono). (Texto 3.NPpc) ${ }^{1}$

La docente se sirve de un juego para hacer la transición entre los ejercicios de una clase de segundo grado de nivel primario. Se trata de una estrategia para ganarse la atención de los niños que, además, le permite organizarlos para el juego o ejercitación siguiente. El tipo discursivo que estructura el enunciado es el discurso interactivo como se comprobará a continuación.

\subsection{Los tipos discursivos}

Los textos presentan modalidades de organización muy variadas, porque con ellos se refieren actividades colectivas humanas extremadamente variables, construidas en el marco de interacciones comunicativas que pueden ser muy diferentes.

Los textos de las clases observadas tienen la finalidad de organizar, clarificar y evaluar acciones generales (Bronckart, 2007) que, en el área de la Educación Física, constituyen, generalmente, acciones motrices. Y están conformados por segmentos mixtos que traducen el mundo ordinario de los agentes (docentesestudiantes), es decir, de la situación comunicativa de la clase. Concretamente, el tipo discursivo discurso interactivo organiza toda la clase de principio a fin y tiene incorporados segmentos de los tipos discurso teórico y relato interactivo, según los diferentes momentos de la actividad de la clase. En efecto, en todos los casos analizados, ambos tipos discursivos se documentan articulados con el discurso interactivo: el discurso teórico aparece en el momento de la formulación y la reformulación de las consignas para guiar la realización de las tareas, mientras que el relato interactivo se constata, generalmente, al final de las clases, momento en el que el docente realiza la recapitulación de lo realizado (para reflexionar y/o evaluar la práctica) y proyecta a cerca de las clases siguientes. 
Por tanto, el docente de educación física para lograr interactuar en la clase se sirve del tipo discursivo discurso interactivo, conformado por segmentos de texto en los que predominan las referencias al agente y los interlocutores que realizan las acciones, que se manifiesta en la presencia de elementos lingüísticos denominados deícticos. Además, en una situación comunicativa cuya finalidad es la realización de acciones con gran contenido técnico, el tipo discurso teórico permitirá dar cuenta de los desarrollos teórico-prácticos que llevarán a la ejecución de una práctica determinada:

P: Golpe de manos bajas no tiene precisión, queda más o menos... Su función es recibir el saque de potencia que viene del otro lado o recibir un [...] La idea es que la pelota quede muerta en el aire para que otro la acomode y un tercer jugador salte y remate. Esa es la idea, no pasarla del otro lado en el primer golpe [...] uno recibe, otro acomoda y el tercero remata. Esa es la idea general del juego. Básicamente es eso el concepto. Con ésta sólo recibo (se refiere a la mano). Yo las posiciones, aquí, ya las marqué. Hacemos eso y jugamos. (Texto 27.NMcb) ${ }^{2}$

La cita contiene segmentos de discurso teórico (subrayado) y de discurso interactivo (negrita).

Entonces, es posible observar que las condiciones concretas de realización de las actividades (Leontiev, 1983) determinan la forma en que elaboramos nuestros enunciados. Las finalidades pretendidas en cada momento de la actividad de la clase llevan al docente a utilizar diversos recursos de la lengua con los que elabora textos que se estructuran en los tipos discursivos más pertinentes y adecuados para garantizar el desarrollo de la clase.

\subsection{Lenguaje desplegado y lenguaje sintético}

El fenómeno verbal expuesto permite numerosas interpretaciones, siempre relacionadas con las condiciones físicas del aula o gimnasio donde transcurren las clases y con el tipo de prácticas que las conforman.

En efecto, tanto el espacio como el tenor de las acciones motrices, determinan que la actividad de la clase sea muy dinámica, lo que implica un determinado uso del lenguaje. El dinamismo, el movimiento y la manipulación de objetos en espacios amplios determinan que las clases de Educación Física cuenten con un elevado uso de lo que hemos dado en denominar lenguaje sintético. Dicha categoría surge frente a la de lenguaje desplegado (Luria, 1984).

La siguiente tabla 1 muestra la articulación de los dos tipos de análisis implementados a través de las categorías elaboradas:

TABLA 1

Relación entre las acciones generales y las acciones verbales

\begin{tabular}{|l|l|l|l|}
\hline \multicolumn{2}{|l|}{ Análisis Textual } & Análisis de la Actividad de Comunicación de los Textos \\
\hline $\begin{array}{l}\text { Usos de } \\
\text { Lenguaje }\end{array}$ & $\begin{array}{l}\text { Tipos } \\
\text { discursivos }\end{array}$ & Momentos de la actividad & Modalidad de la interacción \\
\hline $\begin{array}{l}\text { Lenguaje } \\
\text { desplegado }\end{array}$ & $\begin{array}{l}\text { Discurso } \\
\text { teórico }\end{array}$ & $\begin{array}{l}\text { Estudiantes sentados, } \\
\text { escuchando }\end{array}$ & Formulación de la consigna \\
\cline { 2 - 2 } & $\begin{array}{l}\text { Relato } \\
\text { interactivo }\end{array}$ & & Explicaciones teóricas \\
\cline { 4 - 4 } $\begin{array}{l}\text { Lenguaje } \\
\text { sintético }\end{array}$ & $\begin{array}{l}\text { Discurso } \\
\text { interactivo }\end{array}$ & $\begin{array}{l}\text { Estudiantes realizando los } \\
\text { ejercicios }\end{array}$ & Repetición de la tarea \\
\cline { 4 - 4 } & & Ampliación de la tarea \\
& & Reformulación de la tarea \\
\hline
\end{tabular}

En consecuencia, ambas formas de lenguaje surgen en situaciones comunicativas de la clase muy distintas y tienen una conformación lingüística diferente. 


\subsubsection{Lenguaje desplegado}

El estudio de los textos de las clases mostró la diversidad de formas que contienen los textos analizados y que hemos sistematizarse en dos usos del lenguaje. Por un lado, se documenta un uso desplegado del lenguaje como en cualquier otra área de conocimiento. Este se concentra en dos momentos de la actividad con dos finalidades precisas, primero, la formulación de la tarea y, posteriormente, en la instancia final de la clase, la evaluación de lo realizado y la prospección para futuras sesiones.

Por tanto, el lenguaje desplegado se ha documentado en los momentos de la clase destinados a la formulación de las consignas y a la recapitulación de lo realizado, cuando los alumnos, sentados alrededor del profesor, se recuperan de la práctica física intensa y escuchan al docente. Ambas situaciones comunicativas determinan la construcción de enunciados con formas discursivas elaboradas y complejas respecto del nivel sintáctico de la lengua.

En consecuencia, la organización de los enunciados, por más que sean textos orales, se aproxima al orden escritural y contienen organizadores textuales (subrayados en las siguientes citas) que separan y relacionan los contenidos desarrollados:

Siéntense (los alumnos se sientan alrededor del profesor). Dos o tres cositas para ir corrigiendo en cuanto al juego. Primero, en cuanto al ataque: no me apresuro para buscar a mi compañero para pasar la pelota. La secuencia es: recepciono, miro y, después, paso. No es recepción, paso y, después, miro a ver a quien le pasé la pelota. Entonces, una vez que aseguré la posición de la pelota, aseguro la ubicación de mi compañero y hago el pase. [...] ¿Está bien? Ahora, en cuanto a los que defienden, brazos flexionados y adelante (coloca los brazos). Yo estoy defendiendo... (Texto 21.NMcb) ${ }^{3}$

Chicos, tienen buen juego, lo único... Hay dos cosas fundamentales: primero, lo que nos mata siempre, en todo lo que hacemos, es la duda y más en el deporte, pero en la vida, en general. Cuando dudamos de algo, aunque seamos buenos, por ahí, les sale mal. Y les pasa, a veces tienen un buen golpe, dudan un poco. Hay que tener confianza. Péguenle con confianza, es el momento en el que se tienen que equivocar, tienen que practicar... Entonces, no tengan tanto miedo a pegarle mal y menos en un grupo como éste. El grupo es muy bueno, es tolerante. El grupo a pesar de algunos gritos, algunas cosa, es muy respetuoso tanto de las habilidades como de las [...] de cada uno y eso es lo que hace crecer al grupo como grupo. Por eso todos pueden jugar... (Texto 38.NMco) ${ }^{4}$

Las citas constituyen ejemplos del tipo discursivo relato interactivo, segmentos que presentan una textualización clara y precisa, "conforme a la organización divisible del pensamiento [...] una construcción sintáctica en el sentido de proceso, que se apoya en un verbo" (Jakubinskij, 2018, p. 82).

\subsubsection{Lenguaje sintético}

En el curso de las clases durante la realización de la práctica, se ha registrado un uso del lenguaje breve y sintético. Se trata del empleo reiterado de determinadas palabras, particularmente verbos y sustantivos, en las que se condensa el sentido de todo un enunciado, por lo general, una orden en relación a una acción general (motriz). Este uso corresponde a los momentos de la clase en los que predomina la práctica, y los estudiantes realizan los ejercicios prescriptos.

Es muy común en las clase que, una vez que el profesor ha formulado la consigna y, por lo tanto, prescripto la tarea, en función de lo que los alumnos hagan, tenga que insistir en un aspecto la tarea con el fin de enseñar y/o corregir la técnica. Se trata del proceso por el cual los profesores dirigen las prácticas sin interrumpir el hacer de los alumnos. Es una forma particular de construir la interacción en la clase sin interrumpir la práctica, corrigiendo e instando a continuar la ejercitación, mediante el lenguaje sintético. Los profesores del 
área emplean enunciados breves, a veces muy breves: "pierna”, "más pierna" con los que dan indicaciones respecto de la flexión de piernas para la realización del pase de manos altas en el juego de vóley.

Para ilustrar lo expuesto presentamos, de una clase que tuvo lugar a mitad de curso, en primer lugar, el enunciado de la consigna:

Entonces, empezamos con manos bajas [...] Una pelota cada dos, y hacen lo que ha hecho Nacho: uno la lanza, otro la pega. Una cada dos personas. (Texto 27.NMcb) ${ }^{5}$

En segundo lugar, transcribimos lo que el docente decía durante la práctica, por considerarlo un ejemplo de lenguaje sintético:

P: Más piernas... (le indica a un alumno).

P: Piernas (al alumno que no flexiona las piernas).

P: Más piernas (le dice a un alumno que aunque flexiona, requiere de mayor flexión)

$\mathrm{A}^{\mathrm{o}}$ : Profe.

P: Compensá un poquito más (el profesor mueve el cuerpo y, sin utilizar la pelota, muestra al alumno cómo ha de hacer).

[...]

P: Doblá, doblá por los codos (al tiempo que habla, el profesor coloca los brazos del modo en que indica). (Texto 27.NMcb) ${ }^{6}$

El lenguaje sintético está estrechamente relacionado con la oralidad y el diálogo y determina la estructura de los textos de las clases, haciendo que se organicen en el tipo discurso interactivo. La comprensión mediante esta forma de lenguaje fue posible porque el profesor y los alumnos compartían el espacio físico y el tiempo en el que se producían las acciones verbales, y porque las clases observadas correspondían a la mitad de la cursada. Todo ello favoreció la complicidad y el entendimiento: los estudiantes conocían el sentido de las palabras habituales en la clase, por lo que no fue necesario reponer todo el contexto para hacerse comprender.

El lenguaje sintético está presente en todas las sesiones observadas:

Salen las tres. Salen las tres, [...] Preparadas... Sólo las tres primeras, nada más. ¿Con qué mano agarro? Izquierda. Pasé el primer cono, cambio de mano, trabajo con la derecha; pasé el segundo cono, cambio de mano otra vez, sigo con la izquierda; doy la vuelta y vengo rapidito, derechito para acá. Preparadas... (Texto 35.NM.co $)^{7}$

En otras clases se documentó el empleo de grupos nominales y preposicionales que constituyen prescripciones: "manos al costado", "veinte, trotecito", "por la nariz", "boca abajo, tres de diez, flexiones de brazos". La fragmentación de los enunciados es solo aparente, ya que los alumnos los comprenden y realizan la práctica. Se trata, pues, de un proceso de condensación del pensamiento y simplificación sintáctica del texto para asegurar la consecución de la finalidad pretendida: la ejecución de tareas motrices, el desarrollo de habilidades y la incorporación de la técnica. Un enunciado breve puede ser muy efectivo, si de realizar acciones generales (motrices) se trata.

En síntesis, la presencia de enunciados construidos mediante lo que podríamos denominar sintaxis abreviada (ruptura típica de los estereotipos propios del habla dialogal), es propia del lenguaje oral dialogado. La particularidad de las clases de Educación Física es que los alumnos dialogan con el profesor tanto a través de sus acciones motrices (lo que hacen) como de las verbales. La instancia de la clase presupone el contacto visual de los interlocutores, por lo que es posible registrar la mímica facial y gestual, y escuchar el tono de voz de quien enuncia. Son aspectos que favorecen la comprensión y permiten la comunicación a través de las alusiones, como un complemento de las miradas que se cruzan de los interlocutores (Jakubinskij, 2018). 


\section{Discusión}

La situación comunicativa de los textos de las clases de los que han sido extraídas las citas, corresponde a sesiones dictadas durante el segundo cuatrimestre (agosto-noviembre), lo que propició que el docente y los estudiantes compartieran códigos y cierta complicidad que hizo posible la comunicación. De modo tal que es posible afirmar, citando a Jakubinskij (2018), que las rutinas que se repiten en las clases dan lugar a situaciones estereotipadas. Concretamente, en el momento de la ejercitación se registra un caudal de interacciones verbales estereotipadas relativas a los ejercicios que se realizan.

Asimismo, el proceso de comprensión que realizan los estudiantes no solo es obra de las palabras del profesor, sino, también, de la situación comunicativa: del hecho de que en cada ejercitación se den situaciones similares de enseñanza y aprendizaje. Por ello, es posible afirmar que lo que hemos denominado lenguaje sintético son, en efecto, estereotipos lingüísticos que favorecen la realización de la práctica. Nos referimos al hecho de que el profesor use constantemente las mismas palabras y construcciones sintácticas similares: "Paso la pelota, recibí, un, dos, tres (hace la pinza) pico, un, dos, tres y lanzo" (Texto 21.NMcb) ${ }^{8}$, y un mismo tono de voz en una situación de clase que se repite, hace que esas "frases queden como petrificadas y se transformen en una especie de estereotipos sintácticos complejos" (Jakubinskij, 2018, p. 81).

En consecuencia, aceptamos como una característica común a la interacción en el ámbito educativo de la clase, la presencia del discurso interactivo que contiene y organiza otros tipos discursivos. Y creemos que, particularmente, en el área de la Educación Física la presencia de dicho tipo discursivo y del lenguaje sintético se debe a la necesidad de articular las acciones verbales y las generales: más breve es el enunciado, más efectivo su efecto en el accionar general de los alumnos, y mayor la probabilidad de conseguir el objetivo de corregir y enseñar aspectos en los que los alumnos presentan dificultad.

Consideramos oportuno finalizar con la referencia y una breve interpretación de lo desarrollado por la especialista Bettina Ried. La autora en su estudio sobre el lenguaje verbal de los profesores de Educación Física, menciona la presencia de lenguaje coloquial y lenguaje formal en el aula. Desde el marco teórico del ISD que orienta nuestra investigación, pensamos que lo que identifica como lenguaje coloquial corresponde a los enunciados del tipo discurso interactivo porque, como hemos explicado, contienen marcas (pronombres) relativas a quienes intervienen en la comunicación (al profesor y los alumnos). Mientras que el lenguaje formal que Ried (2017) documenta se correspondería con los segmentos de discurso teórico y de relato interactivo que hemos identificado incluidos en el discurso interactivo de la clase.

Es preciso mencionar, no solo la variedad de formas de lenguaje documentadas, sino el modo en que se articulan y enriquecen la comunicación.

\section{A MOdo DE SÍNTESIS}

En cada momento de la actividad de la clase y según las finalidades pretendidas, se documentan usos específicos de lenguaje, es decir, formas determinadas de construir los enunciados. Podemos afirmar que inconscientemente los docentes recuren a los diferentes tipos discursivos para que la comunicación con los estudiantes sea efectiva. En el caso particular de la Educación Física y como consecuencia de de la especificidad de las prácticas propias del área, las clases suelen ser muy dinámicas, lo que propicia lo que hemos dado en denominar lenguaje sintético.

En otras palabras, se observa que el contexto en el que tienen lugar la clase determina la forma y particularidades de los enunciados elaborados por los docentes de Educación Física. Concretamente, el espacio físico amplio propio de un gimnasio, las características de las tareas (acciones generales) que implican el trabajo en grupos, la utilización de elementos, la disposición de los elementos o materiales en el espacio, 
la distribución de los estudiantes por todo el gimnasio, etc. así como las finalidades pretendidas en cada momento de la clase, dan lugar a formas particulares de expresarse.

El lenguaje es el medio para acceder a los conocimientos específicos del área. La forma de los enunciados de los docentes es consecuencia, y a la vez hace posible, de la constante articulación entre las acciones generales y las acciones verbales que tiene lugar en las clases.

Como puede desprenderse de lo expuesto, es necesaria la articulación de diversos instrumentos de análisis para el estudio de la interacción que genera los procesos de enseñanza en las clases. Después de dos décadas de investigación, llegamos a la conclusión de que el análisis de las consignas de tareas se verá enriquecido con el estudio del diálogo en las clases, tarea que ya hemos iniciado. Convencidas de la necesidad de registrar y analizar lo que se busca enseñar y lo efectivamente enseñado, nos proponemos, pues, continuar y ampliar nuestra línea de investigación.

\section{ReferenCias BibliográficAS}

Bronckart, J.-P. (2004). Actividad verbal, textos y discursos. Por un interaccionismo socio-discursivo. Madrid: Fundación Infancia y Aprendizaje.

Bronckart, J.-P. (2007). Desarrollo del lenguaje y didáctica de las lenguas. Buenos Aires: Miño y Dávila.

Bronckart, J.-P. \& Stroumza, K. (2003). Les types de discours comme traces cristallisées de l'action du langage. En E. Roulet \& M. Burger (Eds.). Les analyses de discours au déft d'un dialogue romanesque. Nancy: PUN.

Goicoechea, M. V. (2015). Las interacciones verbales y las consignas de tareas en Educación Física. Análisis realizado en San Carlos de Bariloche -Patagonia-Argentina. (Tesis de doctorado). Universidad de La Rioja. Edición electrónica. Recuperado de http://dialnet.unirioja.es/servlet/tesis?codigo $=44114$

Goicoechea, M. V. (2019). La comunicación verbal en las clases de Educación Física: Estudio realizado en el marco de la Teoría del diálogo. $13^{\circ}$ Congreso Argentino y $8^{\circ}$ Latinoamericano de Educación Fisica y Ciencias. Facultad de Humanidades y Ciencias de la Educación de la Universidad Nacional de La Plata (Argentina). Recuperado de: http://congresoeducacionfisica.fahce.unlp.edu.ar

Goicoechea, M. V.y Goicoechea M. Á. (2017). Análisis de la actividad registrada en situación de clase: La Educación Física de Nivel Primario en contexto. Poiésis. Revista do Programa de Pós-Graduaçao em Educaçao, 11(20), 433-450. Recuperado de http://www.portaldeperiodicos.unisul.br/index.php/Poiesis/index

Ivanova, I. (2010). El diálogo en la lingüística soviética de los años 1920-1930. En D. Riestra (Comp.), Saussure, Voloshinov y Bajtin revisitados (pp. 45-71). Buenos Aires: Miño y Dávila.

Jakubinskij, L. (2018). Sobre el habla dialogal. Viedma: Editorial de la Universidad Nacional de Río Negro.

Leontiev, A. (1983). El desarrollo del psiquismo. Madrid: Akal.

Luria, A. (1984). Conciencia y lenguaje. Madrid: Visor.

Ried, B. (2017). O profesor de educaçao física "fala bem"? A linguagem verbal usada pero profesor na aula de Educaçao Física e sua adequaçao à especificidades das situaçoes. Revista Pulsar, 9(2), 49-55.

Riestra, D. (2000). Análisis de la enseñanza de la lengua materna desde la teoría de la actividad. En III Conference for Sociocultural Research. Campinas, Brasil [cd-rom].

Riestra, D. (2004). Las consignas de trabajo en el espacio socio-discursivo de la enseñanza de la lengua. (Thèse de Doctorat), Université de Genève. Recuperado de: http://www.unige.ch/cyberdocuments/theses

Riestra, D. (2008). Las consignas de enseñanza de la lengua. Un análisis desde el Interaccionismo socio-discursivo. Buenos Aires: Miño y Dávila.

Riestra, D. (2019). El diálogo y el plurilingüismo en el aula. Revista Chilena de Pedagogía, 1(1), 71-84. 


\section{Notas}

1 El texto 3 corresponde a una clase de Nivel Primario de primer ciclo.

2 El texto 27 corresponde a una clase de Nivel Medio del ciclo básico

3 El texto 21 corresponde a una clase de Nivel Medio del ciclo básico.

4 El texto 38 corresponde a una clase de Nivel Medio del ciclo orientado.

5 El texto 27 corresponde a una clase de Nivel Medio del ciclo básico.

6 El texto 27 corresponde a una clase de Nivel Medio del ciclo básico.

7 El texto 35 corresponde a una clase de Nivel Medio del ciclo orientado.

8 El texto 21 corresponde a una clase de Nivel Medio del ciclo básico. 